\title{
A rare scenario of perforated gastrointestinal stromal tumours of ileum
}

\author{
Mrinal Shankar*, K. Ravindra, Manju R., Radhakrishna Ramchandani
}

Department of General Surgery, All India Institute of Medical Sciences, Raipur, Chhattisgarh, India

Received: 08 July 2020

Accepted: 11 August 2020

\section{*Correspondence:}

Dr. Mrinal Shankar,

E-mail: ms_surg@yahoo.co.in

Copyright: ( ) the author(s), publisher and licensee Medip Academy. This is an open-access article distributed under the terms of the Creative Commons Attribution Non-Commercial License, which permits unrestricted non-commercial use, distribution, and reproduction in any medium, provided the original work is properly cited.

\begin{abstract}
Gastro-intestinal stromal tumours (GIST) are among the common mesenchymal tumours of the gastro-intestinal (GI) tract. It varies in location and presentation. GIST are reported in the stomach frequently (60-70\%), followed by small intestine (20-25\%). Mainly GIST manifest typically with bleeding or vague abdominal pain and discomfort. The spontaneous perforation of GIST is very rare. We report case of a middle-age male patient who presented in emergency with pain in right lower abdomen associated with features of peritonism. After clinical evaluation and preliminary radiological investigations, a working diagnosis of perforated appendix was made. Patient was undertaken for emergency surgery. A diagnostic laparoscopy followed by midline laparotomy was done. Intra-operatively, a perforated and necrotic outpouching at antimesenteric border of terminal ileum was found. Histopathological examination of the resected part of ileum revealed compatibility with GIST. It was strongly positive for cluster of differentiation 117 (CD117) and smooth muscle actin. Patient received adjuvant therapy with Imatinib. A complete surgical resection without extensive lymph node sampling is the primary treatment option. As GIST are rare, a high index of suspicion is warranted for diagnosis and appropriate treatment.
\end{abstract}

Keywords: Gastro-intestinal stromal tumours, Perforation, Terminal ileum, Imatinib

\section{INTRODUCTION}

Gastro-intestinal stromal tumours (GIST) are the common mesenchymal tumours of gastro-intestinal (GI) tract. They are derived from interstitial cells of Cajal, an intestinal pacemaker cell. They can appear anywhere within the gastro-intestinal tract. Although GIST arise most frequently in the stomach $(60-70 \%)$, followed by small intestine (20-25\%). Most GIST manifest typically with bleeding or vague abdominal pain and discomfort. The spontaneous perforation of GIST is rare. They range from small benign tumours to massive lesion with necrosis, haemorrhage and wide metastasis.

There are wide array of investigations, which can be performed pre-operatively including upper GI endoscopy, contrast-enhanced computed tomography (CECT) abdomen and pelvis, endoscopic ultrasound directed fine needle aspiration (EUS-FNA) which requires specialized expertise. In this report, the patient has presented with an acute abdomen, and presentation was masquerading an acute appendicitis, so the above investigations were not performed. Timely surgical intervention was done. Intraoperatively, a perforated tumour was found in ileum. Only after histopathological examination and immunehistochemistry staining, it was identified as GIST. ${ }^{1}$

As GIST are tumours with high risk of recurrence, metastasis and increased disease specific mortality, delay should not be made in treatment. The mainstay of treatment is a complete R0 surgical resection, followed by surveillance and or adjuvant therapy.

\section{CASE REPORT}

A 53-year male with no recognized co-morbid condition, was received in emergency with acute abdominal pain, more confined towards the right lower abdomen for 3 days, 
pain was colicky in nature. Nausea, vomiting and history of fever were not associated. He had unaltered bowel and bladder. The patient had no previous history of any surgery.

On admission, the patient had tachycardia of 108 beats per minute $(\mathrm{bpm})$, hypotensive $(90 / 60 \mathrm{mmHg})$ and afebrile. Physical examination revealed a distended lower abdomen, with tenderness and guarding at right iliac fossa and right lumbar region, no rigidity. Haemogram revealed leucocytosis $\left(13900 / \mathrm{mm}^{3}\right)$ with predominance of polymorphs $(89 \%)$. RFT had raised creatinine $(1.24 \mathrm{mg} / \mathrm{dl})$ and hyponatremia $(124 \mathrm{mEq} / \mathrm{Ll})$, with normal potassium level.

Ultrasonography (USG) whole abdomen with pelvis was suggestive of perforated appendix as tip of appendix was seen to be terminating in a well-defined hypoechoic collection (approximately $6.1 \times 6.4 \mathrm{~cm}$ ) with internal air foci. Surrounding mesentery was inflamed with few subcentimetric lymph nodes in the right iliac fossa. An erect chest $\mathrm{x}$-ray with both domes of diaphragm revealed a thin rim of air under right dome of diaphragm.

Patient was undertaken for emergency surgery under general anaesthesia. A diagnostic aparoscopy was performed first, it revealed dense omental caking and a caked-up lump formed by terminal ileum, appendix and omentum. Laparoscopy was converted to midline laparotomy. After thorough lavage, adhesiolysis and dissection, a perforated and gangrenous diverticulum present at the antimesenteric border of terminal ileum (around $25 \mathrm{~cm}$ proximal to IC junction) was found (Figure 1). Appendectomy, resection and anastomosis of terminal ileum segment was done with a proximal diversion loop ileostomy. Part of omentum was also sent for excision biopsy.

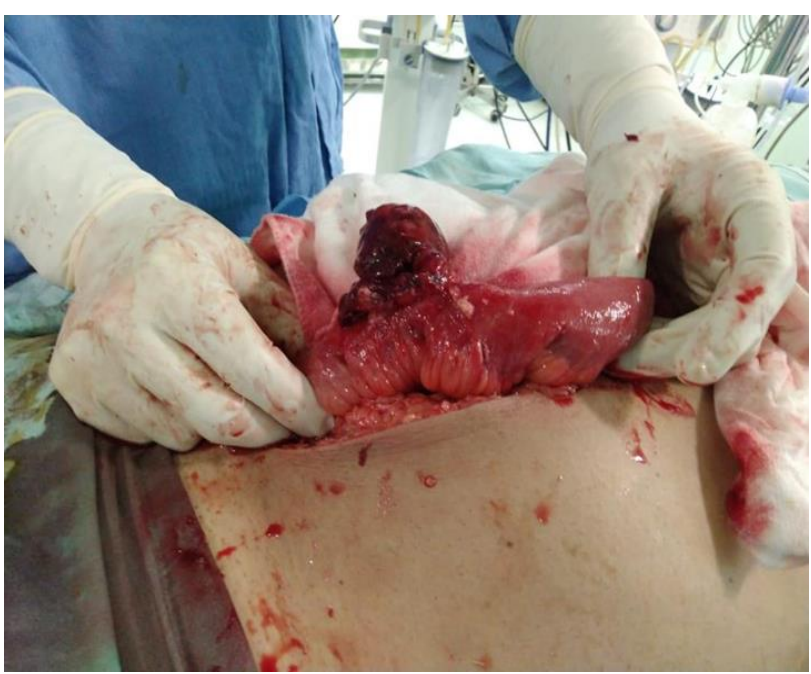

Figure 1: Per-operative finding exposing a necrotic out pouching from the anti-mesenteric border of terminal ileum with perforation at the base.

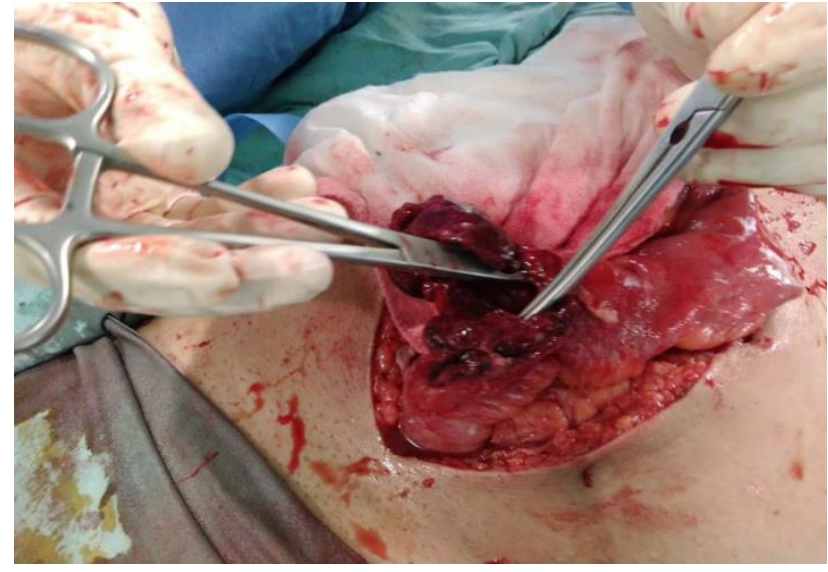

Figure 2: Per-operative demonstration of communication between the perforated tumour and ileum (both proximally and distally).

Histopathological examination of the resected part revealed partially ulcerated ileal mucosa with tumour in sub-epithelium, extending up to muscularis propria, consistent with GIST-intermediate grade. Omentum also showed tumour cells. Mitosis was 5/50 high power fields (hpf). Tumor cells were strongly positive for cluster of differentiation 117 (CD117) and smooth muscle actin (SMA). Cluster of differentiation 34 (CD34) highlighted vessels.

Post-operative period was uneventful, and patient had good recovery. He was started on imatinib. Stoma closure was done after 2 months. Patient is doing well now, and has shown good final outcome of our treatment.

\section{DISCUSSION}

There is no pathognomonic feature of GIST. The symptoms varies with tumour size and more importantly with location. The most common symptom is abdominal pain. Abdominal pain and bleeding into digestive tract (61\% and 58\% respectively) were more frequent in gastric GIST, whereas acute abdominal symptoms were more frequent in jejunal and ileal GIST (40\% and $60 \%$ respectively). ${ }^{2}$

Major emergency presentation of small intestine GIST is intestinal obstruction. ${ }^{3,4}$ However, overt peritonitis caused by GIST rupture is very uncommon. Three types of GIST rupture have been described: closed perforation due to abscess (abscess type), hemoperitoneum due to rupture of the hematoma capsule in the tumour (hemoperitoneum type) and perforation of the digestive tract via a fistula leading to central necrosis of the tumour (bowel perforation type).$^{5}$

In our report, we had a variant of bowel perforation type. There are other interesting case reports of perforated GIST in Meckel's diverticulum and a case of TorricelliBernoulli sign. 6 
GIST are submucosal in origin. Most submucosal GI tumours require surgical resection regardless of histology. Pathologically, GIST have smooth muscle and neuroendocrine features. They are frequently identified by immunohistochemical staining for the C-kit protooncogene (CD117) which is overexpressed in $95 \%$ of these tumors, and for CD34, which is positive in $60 \%$ to $70 \%$ GIST. Other markers that can be demonstrated immunohistochemically and are useful in tyrosine-protein kinase (KIT)-negative GIST are anoctamin-1 (ano-1), platelet-derived growth factor receptor A (PDGFRA), CD 34 , and protein kinase $\mathrm{C}$ theta.

The mainstay of treatment is complete surgical resection, $\mathrm{R} 0$ resection is required. An anatomic resection according to lymph node basins is not required, as lymph node metastases are rare. In the adjuvant setting, patients with c-kit- positive tumours $3 \mathrm{~cm}$ or larger who underwent complete resection and were treated with imatinib for 1 year had a recurrence rate of $8 \%$ compared with $20 \%$ for untreated patients. 7

Our patient was started on imatinib $400 \mathrm{mg}$ once daily for a course of 3 years. A long term follow-up is warranted.

\section{CONCLUSION}

A complete R0 surgical resection without extensive lymph node sampling is the primary treatment option. As GIST are rare, and presents in a multitude of ways, a high index of suspicion is warranted for diagnosis and appropriate treatment.

Funding: No funding sources Conflict of interest: None declared Ethical approval: Not required

\section{REFERENCES}

1. Joensuu H, Eriksson M, Hall KS, Hartmann, Pink D, Schütte J, et al. One vs three years of adjuvant imatinib for operable gastrointestinal stromal tumor: a randomized trial. JAMA. 2012;307(12):1265-72.

2. Menge F, Jakob J, Kasper B, Smakic A, Gaiser T, Hohenberger P. clinical presentation of gastrointestinal stromal tumors. Visceral Med. 2018;34(5):335-40.

3. Caterino S, Lorenzon L, Petrucciani N, Iannicelli E, Pilozzi E, Romiti A, et al. Gastrointestinal stromal tumors: correlation between symptoms at presentation, tumor location and prognostic factors in 47 consecutive patients. World J Surg Oncol. 2011;9:13.

4. Sorour MA, Kassem MI, Ghazal Ael H, El-Riwini MT, Abu Nasr A. Gastrointestinal stromal tumors (GIST) related emergencies. Int J Surg. 2014;12:26980.

5. Sato K, Tazawa H, Fujisaki S, Fukuhara S, Imaoka $\mathrm{K}$, Hirata $\mathrm{Y}$, et al. Acute diffuse peritonitis due to spontaneous rupture of a primary gastrointestinal stromal tumor of the jejunum: a case report. Int J Surg Case Rep. 2011;39:288-92.

6. Hosamani IR, Kodaganur S, Chaukimath SM. Perforated gastrointestinal stromal tumor of the Meckel's diverticulum: a case report and review of literature. Ind J Surg. 2016;78(5):390-5.

7. Tajima T, Nishi T, Tomioku M, Ogimi T, Chan LF, Okazaki T, et al. Perforated gastrointestinal stromal tumor in the small intestine: a rare case of TorricelliBernoulli sign. Mol Clin Oncol. 2018;9(4):399-402.

Cite this article as: Shankar M, Ravindra K, Manju R, Ramchandani R. A rare scenario of perforated gastrointestinal stromal tumours of ileum. Int Surg J. 2020;7(9):3117-9. 